\title{
Numerical and experimental investigation of heat transfer in a solar receiver with a variable aperture
}

\author{
Mostafa Abuseada ${ }^{a}$, Nesrin Ozalp ${ }^{\mathrm{a}, *}$, Cédric Ophoff ${ }^{\mathrm{b}}$ \\ a University of Minnesota Duluth, Mechanical Engineering Department, Duluth, MN 55812, USA \\ ${ }^{\mathrm{b}} \mathrm{KU}$ Leuven, Mechanical Engineering Department, Leuven 3001, Belgium
}

\section{A R T I C L E I N F O}

\section{Article history:}

Received 4 June 2018

Received in revised form 27 August 2018

Accepted 27 August 2018

Available online 4 September 2018

\section{Keywords:}

Solar receiver

Radiation heat transfer

Optical analysis

Variable aperture

Monte Carlo ray tracing

\begin{abstract}
A B S T R A C T
Variable aperture can assist in maintaining semi-constant temperatures within a receiver's cavity under transient solar loading. An in-house code has been developed to model a receiver and effectively control its components to achieve semi-constant temperatures under transients. The code consists of a full optical analysis performed via the Monte Carlo ray tracing method in addition to a transient two-dimensional heat transfer analysis. The system studied consists of a cavity type solar receiver with 60 mm radius fixed aperture on the cavity body, a variable aperture mechanism mounted on the receiver's flange, and a $7 \mathrm{~kW}$ Xenon arc solar simulator. A composite shape consisting of a hemisphere attached to a cylinder is proposed to model the Xenon arc. The in-house code has been experimentally validated through experimental tests for different input currents to the solar simulator, volumetric flow rates, and aperture's radii. The optical analysis was validated based on heat flux measurements, where it had percentage errors of 0.8 , $0.5,1.1$, and $3.2 \%$ for the peak power, total power, half width, and half power. For the heat transfer model, percentage errors of $3.2,2.9$, and $5.3 \%$ at the inlet, center, and outlet sections of the receiver were determined for different flow rates using maximum input current and opening radius. The aperture mechanism was capable of maintaining an exhaust temperature of $250^{\circ} \mathrm{C}$ based on actual Direct Normal Irradiance data. Results showed that the variable aperture is a promising apparatus even in applications where the maximum temperatures are desired based on an observed optimum radius of $57.5 \mathrm{~mm}$.
\end{abstract}

(c) 2018 Elsevier Ltd. All rights reserved.

\section{Introduction}

Current energy production methods adversely affect the environment and lead to global warming. In the United States, an average of 426 million metric tons of carbon dioxide was emitted per month, as of 2017. This emission was mainly from petroleum, coal, and natural gas, where these energy sources represented $78.8 \%$ of the primary energy production [1]. The shift towards renewable energy, especially solar energy, saw its biggest increase starting in 2011. However, there are limitations and challenges of present solar thermal technologies.

A large portion of the solar energy sector consists of Concentrating Solar Power (CSP) plants. These plants implement the use of reflecting mirrors or heliostats in order to focus solar radiation from the sun onto a point or line. The main types of CSP technologies are: parabolic dish, parabolic/enclosed trough, linear Fresnel reflector, and solar tower, with the parabolic trough being the most commonly used technology. However, solar towers with central

\footnotetext{
* Corresponding author.

E-mail address: nozalp@d.umn.edu (N. Ozalp).
}

receivers are gaining much greater attention due to their efficiencies at elevated temperatures enabling implementations in thermochemical processes in addition to direct electricity generation [2]. A solar tower central receiver is a cavity with an aperture that aims for maximizing the irradiation captured while minimizing the re-radiation lost within the cavity [3]. The captured radiation provides high temperature heat that can be used in several applications, including production of direct electricity [4], fuels [5,6], metals [7], and other products [8].

One of the main challenges for solar reactors is due to the transient nature of solar irradiance intercepted by the Earth's surface. This inconsistency of irradiance significantly affects the performance of solar reactors, which require semi-constant temperatures within their cavity to maximize the process's efficiency. There are several notable solar reactor designs in literature [7-10], which aim at investigating this challenge and proposing improvements for design concepts and operating conditions. Most of these designs incorporate the use of an optimum fixed aperture size that is determined through a compromise between maximizing irradiation captured while minimizing re-radiation lost through the aperture for the anticipated working conditions [3]. However, since 


$\begin{array}{ll}\text { Nomenclature } \\ A & \text { area } \\ C & \text { geometry constant } \\ c_{p} & \text { specific heat capacity } \\ D & \text { distribution factor } \\ d & \text { diameter } \\ f & \text { focal length } \\ h & \text { convection heat transfer coefficient } \\ k & \text { thermal conductivity } \\ m & \text { mass } \\ \dot{m} & \text { mass flow rate } \\ N & \text { number of rays } \\ n & \text { number of total surfaces } \\ \hat{n}_{s} & \text { ideal normal vector } \\ \hat{n}_{s}^{\prime} & \text { real normal vector } \\ \dot{Q} & \text { heat rate } \\ \mathcal{R} & \text { random number between zero and one } \\ r & \text { radius } \\ s & \text { arbitrary number greater than zero } \\ T & \text { temperature } \\ t & \text { time } \\ \hat{t} & \text { tangent vector } \\ U & \text { uncertainty }\end{array}$

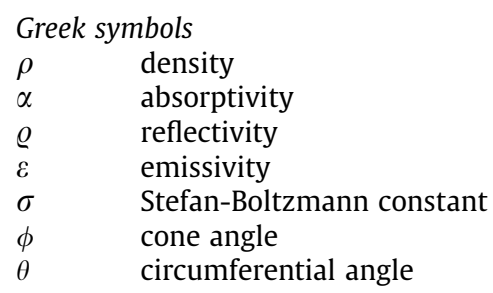

\begin{tabular}{ll}
\multicolumn{2}{l}{ Subscripts } \\
ap & aperture \\
$c$ & cavity \\
$c d$ & conduction \\
$c n v$ & convection \\
ex & exhaust \\
$i, j, l, m$ & element number \\
in & incident \\
$n$ & set number \\
$r$ & reflected \\
$r d$ & radiation \\
$s$ & specular error \\
$t r$ & truncated
\end{tabular}

these reactors have fixed apertures, they are not able to compensate for large fluctuations in the irradiance without sacrificing other aspects of the process. The most commonly used approach to maintaining semi-constant temperatures within the solar reactor is adjusting the feedstock's flow rate to compensate for any irradiance fluctuations [11,12]. However, this approach disturbs the flow dynamics and is usually not feasible in processes where the flow pattern has to be preserved. Furthermore, it changes the production rate.

A promising approach to compensate for the fluctuation in solar irradiance is the implementation of a variable aperture mechanism instead of a fixed one. Such aperture will then be capable of maintaining semi-constant temperatures within the solar reactor in applications where controlling the feedstock's flow rate fails to do so. The purpose of the mechanism is to act just like the iris of an eye, where it expands when the irradiance is lower than that required and contracts when it is higher. Thus, compensating for any irradiance fluctuation without the need to disturb the flow pattern. Various aperture mechanisms have been developed with different designs and approaches to make this mechanism much more effective and sustainable for solar reactors [13-15].

To be able to effectively control the aperture mechanism in addition to other components within the solar receiver, such as the feedstock's flow rate, the receiver needs to be modeled thoroughly. Therefore, an in-house code has been developed to model the receiver, which consists of a full optical analysis of the system as well as a transient two-dimensional heat transfer analysis. The modeled system consists of a solar receiver, variable aperture, and a solar simulator with a Xenon arc bulb. There are several methods that can be incorporated to conduct an optical analysis, such as the Radiosity Net Exchange (RNE) and the Monte Carlo Ray Tracing (MCRT) methods [16-18]. However, the MCRT is the most commonly used method to simulate thermal radiation due to its capability of incorporating probabilistic density functions and other surface properties that the RNE method cannot incorporate [19-21]. Therefore, the MCRT technique has been utilized to perform the optical analysis of the system.

The optical analysis begins by modeling the solar simulator and its Xenon arc to obtain the heat flux distribution and direction of the source's incident radiation into the receiver's aperture. The arc has been previously modeled as a cylindrical volume, which is mainly based on manufacturer specifications [9,22]. However, it was illustrated that the arc cannot be accurately defined and modeled by a single simple emitting shape, whether it is a sphere or a cylinder. A concentric multilayer model of the arc that incorporates both of the previously stated geometries is capable of capturing the complex characteristics of the arc [23]. Therefore, this study proposes the use of a composite shape that consists of a sphere and a cylinder, as discussed later. Once the Xenon arc source has been modeled, the optical analysis progresses to simulate the incident irradiance from the source to the solar receiver in addition to any reflections and re-radiation within the receiver's cavity to obtain the distribution of power. By then, the optical analysis concludes and the model advances to the heat transfer analysis, where it has been performed using the finite volume method due to its capability of strictly abiding to all conservation laws [24]. These two analyses are usually coupled together when an in-depth study of radiative heat transfer is necessary [25].

\section{System description and experimental setup}

The system consists of three main components: a cavity type solar receiver, variable aperture, and $7 \mathrm{~kW}$ high-flux solar simulator that acts as a radiative source. At this experimental stage, the fluid flow through the receiver is Nitrogen. The details of each component are further discussed in the following sections, while the overall experimental setup in the lab is shown in Fig. 1.

\subsection{Solar receiver}

This is a cavity type solar receiver that is made of a $200 \mathrm{~mm}$ hollow cylinder with an inner radius of $60 \mathrm{~mm}$ and a thickness of $15 \mathrm{~mm}$. It has front and back plates bolted onto it to form the receiver's cavity. The front plate is $160 \mathrm{~mm}$ in radius and has a thickness of $25 \mathrm{~mm}$, while the back plate is $75 \mathrm{~mm}$ in radius and has a thickness of $15 \mathrm{~mm}$. All of the receiver's components are made of stainless steel 316 . The cavity is sealed with a $3.175 \mathrm{~mm}$ thick quartz 


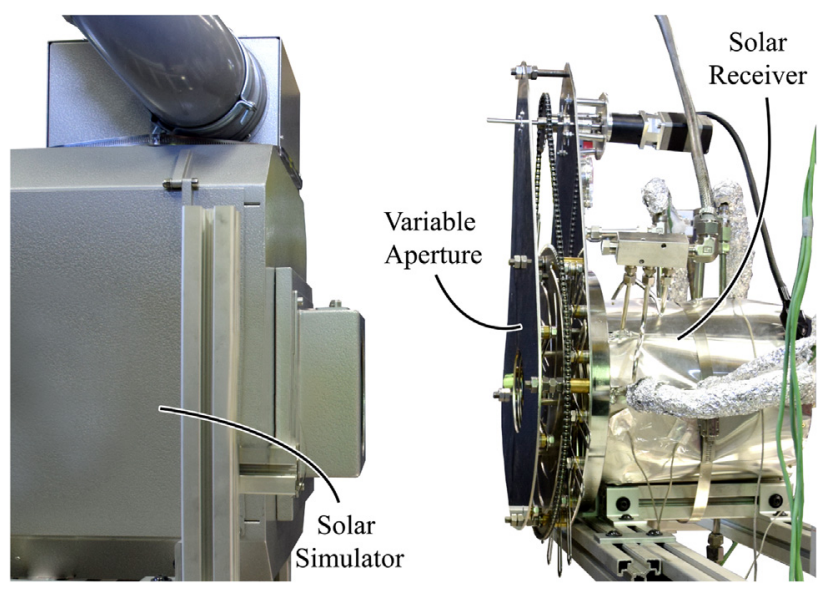

Fig. 1. Experimental setup in the lab.

window at the front side, which allows for the entrance of the solar irradiance. The variable aperture is then attached to the front plate in order to control the irradiance into the receiver.

The receiver has three tangential inlet ports at the front side for fluid flow entry into the receiver. The tangential inlet ports are circumferentially distributed with equal angles of $120^{\circ}$ in order to create a vortex inside the cavity and enhance heat transfer. The receiver also features 11 thermocouple ports for measuring the temperature distribution across the cavity and at the exhaust using type $\mathrm{K}$ thermocouple probes and one outlet port for fluid flow at the back side. In order to insulate the solar receiver, it is covered by $40 \mathrm{~mm}$ of ceramic fiber (Cerablanket AC2) provided by Morgan Thermal Ceramics. An illustration of the CAD model is shown in Fig. 2.

\subsection{Variable aperture}

Similar to a flow control valve, the variable aperture serves as an orifice to regulate irradiation. As the irradiance at the aperture increases, the orifice will contract, whereas it will expand to compensate for lower irradiance levels. This ultimately allows to control the receiver's temperature according to the volatile weather conditions and variation of the Sun's position. The variable aperture, also called the iris mechanism relating to the way it mimics the iris of the human eye, is shown in Fig. 2. A set of eight flat blades are actuated by a high-resolution stepper motor through chain transmission. The multi-planar configuration allows to translate each blade over a distance of $70 \mathrm{~mm}$, which forms a continuously changing aperture size. The aperture diameter ranges from 0 to $100 \mathrm{~mm}$, completely closed and open respectively, and smooth transition is guaranteed by the geometry of the blades and the disc actuating them. Three aperture sizes of the mechanism are shown in Fig. 2. Further details about the geometric constraints, torque calculation, and other aspects of the design methodology can be found elsewhere [26].

\subsection{Solar simulator}

This is a simulator provided by Proyecson that consists of a $7 \mathrm{~kW}$ Xenon arc high discharge lamp with an ellipsoidal reflector. The reflector has a truncated diameter $\left(d_{t r}\right)$ of $340 \mathrm{~mm}$ and two focal points $\left(f_{1}\right.$ and $\left.f_{2}\right)$ at distances of 55 and $825 \mathrm{~mm}$ from the reflector. The Xenon arc lamp is powered by a modulated DC supply generated from an AC/DC rectifier and the simulator is current controlled within the range of 115-155 A in order to vary the output power. The simulator also has an air extraction by a centrifuge axial fan at $655 \mathrm{~m}^{3} / \mathrm{h}$.

\subsection{Optical characterization of the solar simulator}

To model the system accurately, it is vital to quantify the power intercepted by the solar receiver as well as identify the radiation intensity distribution. Therefore, the power irradiance at the second focal point was experimentally characterized through a heat flux measurement technique. The method implemented is an indirect flux mapping technique, since it is one of the most commonly used methods due to its high data acquisition and spatial resolution [27]. The method incorporates the use of a heat flux gage, an $80 \times 80 \mathrm{~mm}^{2}$ Lambertian target, a Complementary Metal Oxide Semiconductor (CMOS) or a Charge-Coupled Device (CCD) camera, and optical filters. Also, an XYZ slider is used to accurately control the position of the target at the focal plane, with steps of $0.08 \mu \mathrm{m}$ in three different axes. Further details of this setup and methodology can be found elsewhere [26].

The experimental optical characterization of the solar simulator was performed for three different input current values of 115, 135, and $155 \mathrm{~A}$. The three-dimensional heat flux distribution on the $80 \times 80 \mathrm{~mm}^{2}$ target placed at the second focal point is shown in

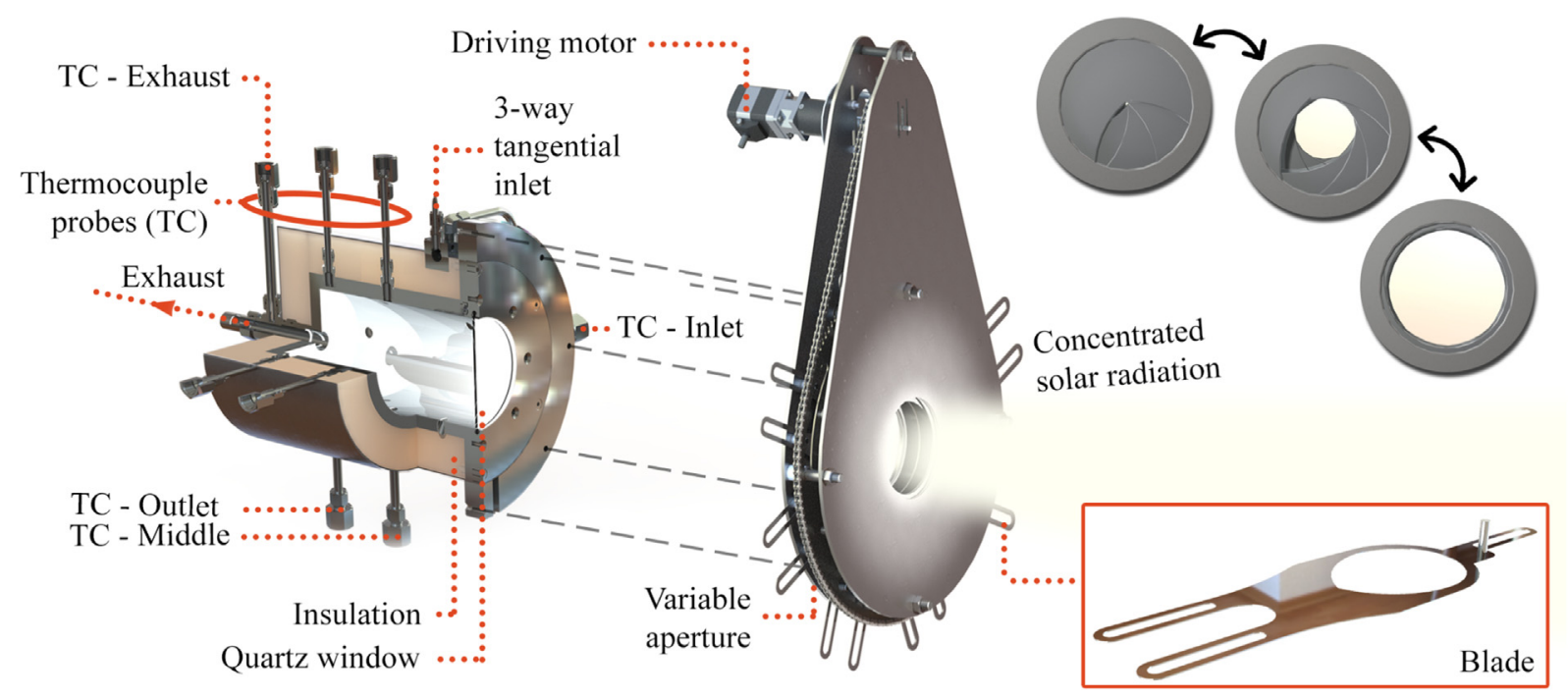

Fig. 2. CAD model of the solar receiver and variable aperture. 


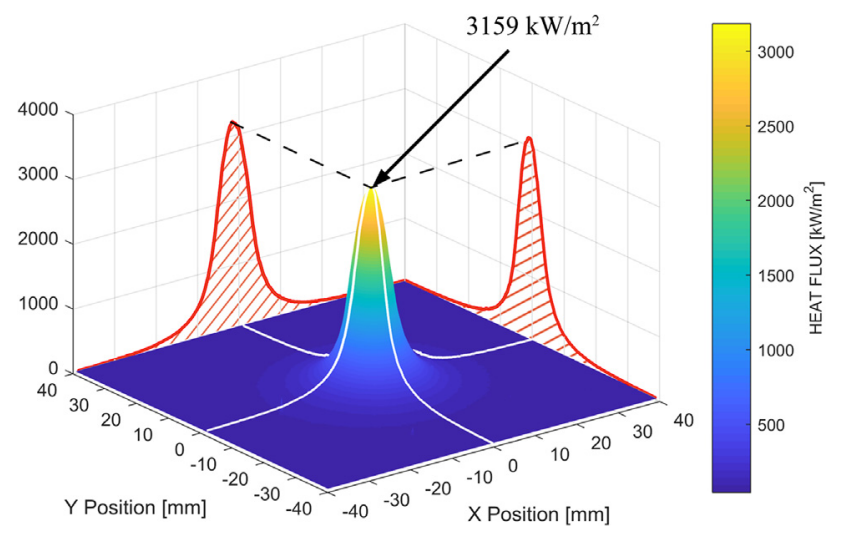

Fig. 3. Three-dimensional heat flux distribution at $f_{2}$ for $155 \mathrm{~A}$.

Fig. 3 for the maximum current value, where the maximum heat flux at the center of the target is indicated. As it can be noticed, the heat flux distribution of the solar simulator exhibits bivariate normal distribution. For the input current range of 115-155 A, the maximum heat flux obtained ranges from 1776 to $3159 \mathrm{~kW} / \mathrm{m}^{2}$, while the total power intercepted ranges from 563 to $903 \mathrm{~W}$. Also, the half width, defined as the radial distance from the distribution's peak to that of half its value, has been determined to range from 4.6 to $4.7 \mathrm{~mm}$ for the entire current range. Finally, the half power, defined as the radial distance from the center in which half of the power on the target is contained in, has been determined to range from 3.75 to $3.85 \mathrm{~mm}$. This clearly indicates that the power of the simulator has a more pronounced effect on the peak of the flux distribution as opposed to its overall shape and characteristics.

\section{Numerical methods}

The full numerical model consists of various analyses to simulate the solar receiver accurately. The model starts with an optical analysis by incorporating the MCRT method to simulate the solar simulator and distribution of power from the radiation source to the receiver's cavity. It also simulates power distribution of the re-radiation emitted within the cavity and determines any radiation losses. The optical analysis is then coupled with the heat transfer analysis performed per finite volume method, which is two-dimensional in the solid phase and one-dimensional in the fluid phase.

\subsection{Model description and discretization}

Prior to describing the model any further, it is vital to state the assumptions that the numerical model incorporates. Since all system components are placed on a single axis and their geometries are symmetrical, the system can be assumed to be twodimensional without sacrificing the accuracy of the model. The assumptions for each analysis are listed as follows.

For the optical analysis, the assumptions are:

- The analysis is three-dimensional

- All surfaces are diffuse gray and have surface properties that are independent of temperature

- The fluid is a non-participating medium

- The quartz window has a refractive index of one

However, for the heat transfer analysis, the assumptions are:

- System is two-dimensional in the solid phase and onedimensional in the fluid phase

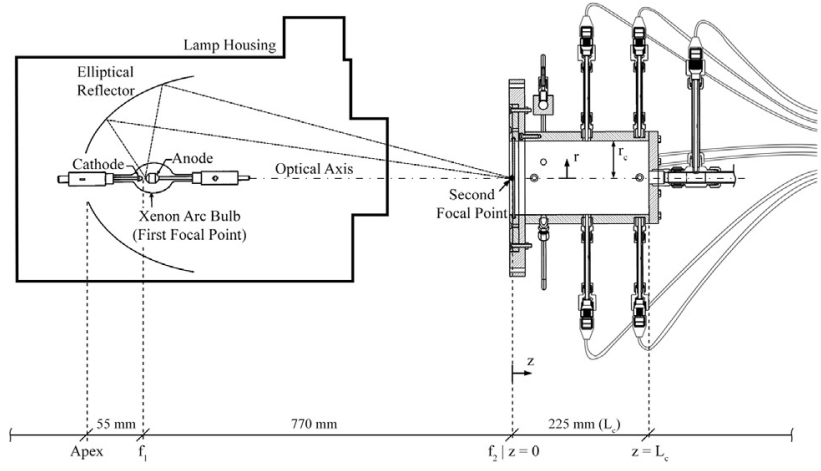

Fig. 4. Overall schematic of the system.

- Fluid enters from inlet ports to receiver's cavity right at the quartz window and then progresses throughout

- Variable aperture's temperature does not affect the heat transfer analysis

Per aforementioned details and assumptions, the best choice for a coordinate system is Cartesian for the optical analysis and cylindrical for the heat transfer analysis. Since the heat transfer is the main driving analysis, the results from the MCRT should be modified to be compatible with the cylindrical system. A schematic of the overall system is shown in Fig. 4, where the origin is placed at the second focal point. Based on the overall system illustrated in Fig. 4 as well as the assumptions, the system will be discretized in the form of annulus elements throughout the solid phase and disk-shaped elements throughout the fluid phase.

\subsection{Optical analysis}

Very few analytical solutions exist for thermal radiation problems, even in the presence of a non-participating medium [17]. Therefore, the MCRT method is usually employed in order to analyze these situations, where the behavior of the system cannot be easily or accurately determined. The method functions by dividing up a radiation source into a large number of energy bundles of equal power values, referred to as rays, where random numbers are incorporated to emerge rays from the radiative source in a stochastic manner [18]. Each emerged ray is then traced as it interacts with different surfaces of an enclosure, where the loss of the ray from the enclosure is a possibility. The outcome of the MCRT method is the distribution of power from radiative sources and surfaces, and so, it incorporates the use of a distribution factor, $D_{i j}$. This factor represents the ratio of absorbed radiation by surface $j$ to that emitted by surface $i$ due to direct radiation and all possible reflections [18].

Since this method relies heavily on large amounts of rays and use of random numbers, at least several millions of random numbers need to be drawn very rapidly to make this method computationally feasible. The solution of using an externally determined actual set of random numbers would need a massive amount of computer storage [17]. Therefore, the use of pseudorandom numbers has been incorporated. These numbers are predetermined values that seem to be random, but not truly random [16]. The use of pseudorandom numbers in the MCRT is a valid alternative when care is taken to evaluate the randomness of these numbers based on the quality of the number generator and the choice of a starting point. In an attempt to decrease the amount of random numbers and rays required, the energy partitioning method can be implemented to make the model more efficient. However, this method is only necessary in open configurations and/or highly reflective 
surfaces [17]. Therefore, it was not implemented into this model. A flow chart illustrating the steps involved in the MCRT method is shown in Fig. 5, which is also known as the forward Monte Carlo method [16].

Before starting with the MCRT, it is important to define all surfaces within the system to be analyzed. The overall schematic is illustrated in Fig. 4, where it will be used to define the surface equations. The equations of the main system components are defined as follows.

Ellipsoidal reflector:

$\frac{\left(z+C_{3}\right)^{2}}{C_{1}^{2}}+\frac{y^{2}}{C_{2}^{2}}+\frac{x^{2}}{C_{2}^{2}}-1=0 \quad z \leqslant C_{4}$

Aperture:

$\sqrt{x^{2}+y^{2}} \leqslant r_{a p} \quad z=0$

Cavity:

$x^{2}+y^{2}-r_{c}^{2}=0 \quad 0 \leqslant z<L_{c}$

Back plate:

$r_{e x} \leqslant \sqrt{x^{2}+y^{2}} \leqslant r_{c} \quad z=L_{c}$

where $\quad C_{3}=\left(f_{2}-f_{1}\right) / 2, \quad C_{1}=C_{3}+f_{1}, \quad C_{2}=\sqrt{C_{1}^{2}-C_{3}^{2}}$, $C_{4}=-C_{3}-\sqrt{C_{1}^{2}\left(1-d_{\text {trunc }}^{2} / 4 C_{2}^{2}\right)}$, and $L_{c}=225 \mathrm{~mm}$.

Once all surfaces within the system have been defined, it is then necessary to define the Xenon arc. As discussed earlier, the arc cannot be accurately modeled by a single simple emitting shape. Therefore, this study proposes a composite shape, placed at the first focal point, consisting of a hemi-sphere with a radius of $0.35 \mathrm{~mm}$ attached to a cylinder with a radius of $1.0 \mathrm{~mm}$ and length

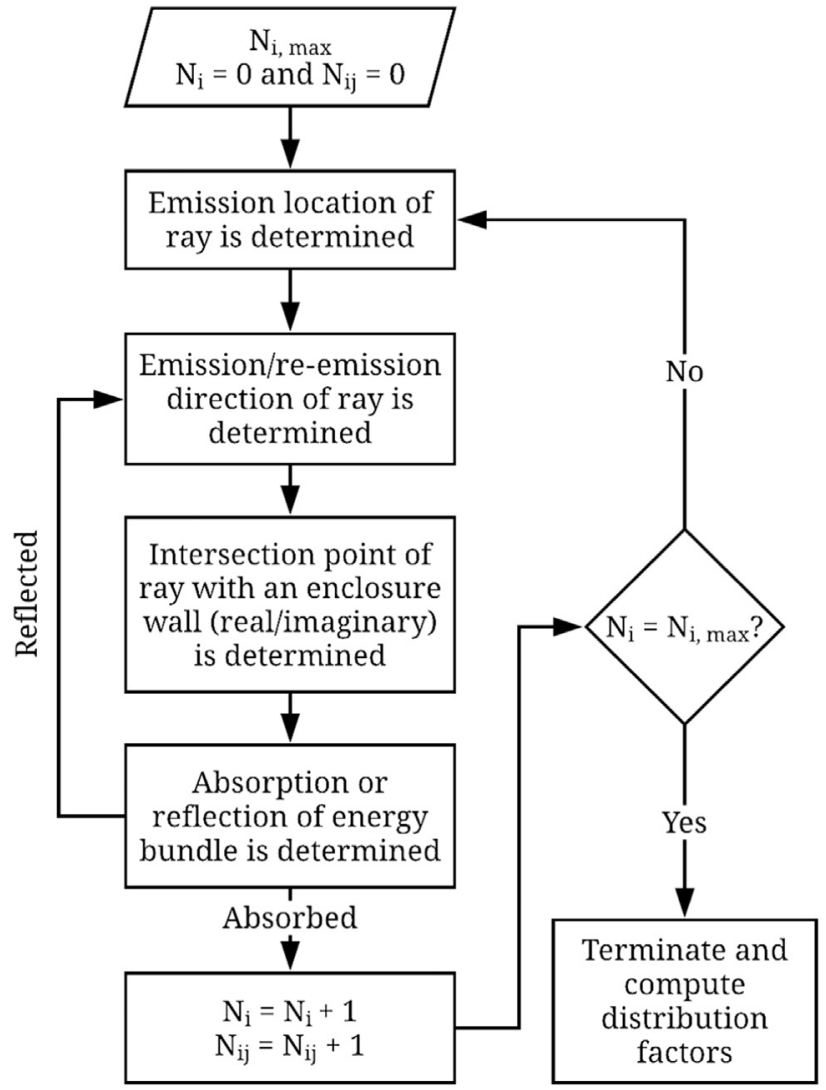

Fig. 5. Flow chart illustrating the MCRT algorithm. of $6.0 \mathrm{~mm}$. The power of the Xenon arc is distributed among these two shapes with a ratio of $2: 3$. It is then possible to proceed with the MCRT algorithm illustrated in Fig. 5, which has to be performed for each emitting source or surface $i$ within the system.

The first step in the MCRT is to equally divide the emitting source or surface into a large number of rays $N_{i}$ and to initialize the counters $N_{i}$ and $N_{i j}$ by equating them to zero. The Xenon arc is treated as previously discussed with its power measured experimentally, while the power of other emitting discretized surfaces is calculated using the Stefan-Boltzmann equation $\dot{Q}_{i}=\varepsilon_{i} A_{i} \sigma T_{i}^{4}$. The number of rays to be used depends on the computational time and accuracy required. A value of at least 10,000 multiplied by the number of surfaces in the analysis is generally recommended [18]. However, a convergence analysis should always be performed to ensure that the results converge to an acceptable accuracy. Based on the convergence analysis, the value of $N_{i}$ used throughout is 20,000 , where this value is in the higher end of the range found in other studies $[19,20,28]$.

The second step is to determine the ray's location of emission. This is performed through implementing the use of the spherical coordinate system along with uniformly distributed random numbers between zero and one. The random numbers are represented as $\mathcal{R}_{n}$, where $n$ represents a set number that should be different for every instance random numbers are incorporated. Since the surfaces are assumed to be diffuse gray surfaces, the wavelength of the ray is not considered and the ray has an equal chance of being emitted from any location and direction within the surface. With that being said, Eqs. (5) and (6) are used to determine the location of emission, depending on the geometrical shape of the emitting surface.

$$
\begin{aligned}
& (r, \theta)=\left(r_{i} \sqrt{\mathcal{R}_{n}}, 2 \pi \mathcal{R}_{n}\right) \\
& (\phi, \theta)=\left(\sin ^{-1} \sqrt{\mathcal{R}_{n}}, 2 \pi \mathcal{R}_{n}\right)
\end{aligned}
$$

The third step is to determine the ray's direction of emission, reemission, or reflection. This is also performed through a similar manner as that defined in the second step by using Eq. (6), since the probability density function is equally distributed along a surface. Having the location and direction of the ray determined, the path of the ray can then be calculated as shown in Eq. (7) and illustrated in Fig. 6a.

$\hat{r}_{r}=\widehat{O P}_{r}+s \hat{d}_{r}$

where $s$ is an arbitrary number, greater than zero, to be calculated in order to find the exact path of the ray.

The above methodology is used in order to determine the direction of all rays, except those reflected by the ellipsoidal reflector. For the reflector, the law of reflection is implemented. Since the law of reflection assumes that surfaces are ideal, the law must be modified to accurately represent real surfaces. This is performed through the introduction of a specular error, in the form of a deviating cone angle $\phi_{s}$ [9]. This angle is illustrated in Fig. 6b, where the real surface normal, $\hat{n}_{s}^{\prime}$, is calculated using Eq. (8). Since a deviating
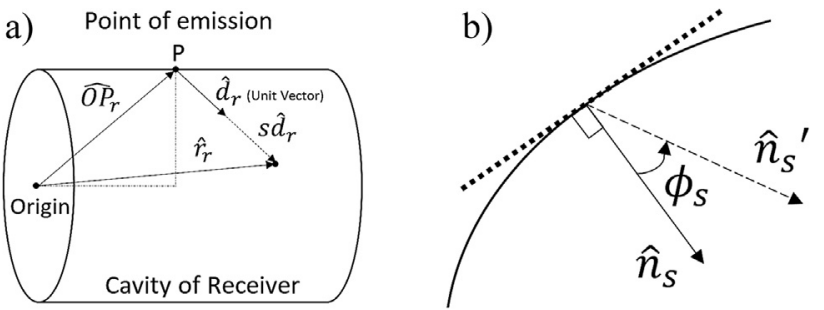

Fig. 6. Illustration of a. path of ray, b. specular error. 
cone angle is introduced, a circumferential angle, $\theta_{s}$, needs to accompany it to fully define the real normal as shown in Eq. (8).

$\hat{n}_{s}^{\prime}=\cos \phi_{s} \hat{n}_{s}+\sin \phi_{s} \cos \theta_{s} \hat{t}_{1}+\sin \phi_{s} \sin \theta_{s} \hat{t}_{2}$

where $\phi_{s}$ has a normal distribution with a mean value of zero and a standard deviation of $6.5 \mathrm{mrad}$, while $\theta_{s}$ has a uniform distribution calculated by $\theta_{s}=2 \pi \mathcal{R}_{n}$. The value for the standard deviation of $\phi_{s}$ lies between the acceptable range examined by Krueger for a similar ellipsoidal reflector [9]. $\hat{t}_{1}$ and $\hat{t}_{2}$ are any two vectors that satisfy $\hat{t}_{1} \cdot \hat{n}_{s}=0$ and $\hat{n}_{s} \times \hat{t}_{1}=\hat{t}_{2}$. Once the real normal is calculated using Eq. (8), the direction of the ray can be calculated using Eq. (9) to find the path of the reflected ray using Eq. (7).

$\hat{d}_{r}=\hat{d}_{i n}-2 \hat{n}_{s} \prime\left(\hat{d}_{i n} \cdot \hat{n}_{s}^{\prime}\right)$

The fourth step is to determine the point of intersection of the ray with an enclosure surface. This is determined using the path of the ray per Eq. (7) as well as the shape and location of the surface defined by one of the equations earlier. The methodology taken for this step will only be described for the receiver's cavity, where other system components undergo a similar procedure.

To determine the intersection with the cavity, the components of Eq. (7) are substituted into the surface Eq. (3). The equation is then expanded and rearranged. This results in Eq. (10), where $s$ can then be solved for. If a value can be calculated where $s>0$, the $\mathrm{x}, \mathrm{y}$, and $\mathrm{z}$ components are calculated using Eq. (7). Then, if the $\mathrm{z}$ position lies within the range of the cavity, the ray is determined to intersect the cavity and the location of intersection has been already determined. This location is then translated to correspond to the specific discretized surface $j$. However, if $s \leqslant 0$ or the $\mathrm{z}$ component does not lie within the constraint, the ray does not intersect the cavity.

$s^{2}\left(d_{x}^{2}+d_{y}^{2}\right)+s\left(2 O P_{x} d_{x}+2 O P_{y} d_{y}\right)+\left(O P_{x}^{2}+O P_{y}^{2}-r_{c}^{2}\right)=0$

In a similar manner, equations representing all surfaces are constructed and used in the same procedure described earlier. If an intersection cannot be determined with any of the surfaces, the ray intersects an imaginary surface out of the system of concern and the ray is deemed lost. $N_{i}$ is then incremented by one, and history of the ray is terminated, where the analysis goes back to step two. However, if an intersection is determined, the analysis proceeds to the next step.

The fifth and final step is to determine whether the ray is absorbed or reflected upon intersection with a surface. This is performed through the use of random numbers, where a generated number is then compared to the properties of the emitting surface. In the case of an opaque surface, $\varrho=1-\alpha$, where $\alpha=\varepsilon$ as per Kirchoff's law for diffuse gray surfaces [16]. The reflectivity used for the ellipsoidal reflector is 0.9 while for the stainless steel receiver is 0.2 . If $\mathcal{R}_{n} \leqslant \varrho$, the ray is deemed to be reflected, and the analysis goes back to step three. There is no need to determine the point of emission, since this is the same as the intersection location. Otherwise, if $\mathcal{R}_{n}>\varrho$, the ray is determined to be absorbed and both parameters $N_{i}$ and $N_{i j}$ will be incremented by one, where the history of the ray is terminated. Then, the analysis goes back to step two and loops in a similar manner, until the value of $N_{i}$ reaches the maximum number of rays specified, where the analysis is then terminated. Finally, the distribution factor will be computed using $D_{i j}=N_{i j} / N_{i}$ and the algorithm moves to the next surface. Upon completion of all surfaces, the complete distribution factor is obtained and it is then possible to proceed to the heat transfer analysis.

\subsection{Heat transfer analysis}

The heat transfer analysis of the solar receiver is performed using the finite volume method, which couples the MCRT method described earlier. Based on the assumptions previously stated, the heat transfer model is two-dimensional in the solid phase and onedimensional in the fluid phase. With that being said and taking into account the geometry of the solar receiver, the control volumes for the solid phase are small annulus elements while they are small disk-shaped elements for the fluid phase. The resulting mesh of such a discretization in addition to the control volume elements are shown in Fig. 7.

For the solid phase, conservation of energy is used in order to determine the governing equation for each volumetric element, which describes the transient heat transfer of the entire model. Each group of elements has a different governing equation, depending on their location within the receiver. The most critical location with the most heat transfer occurring is the first layer within the cavity walls. Therefore, the derivation analysis will be performed for the group of elements that lie within the horizontal cylinder portion of the cavity. For this, the conservation of energy is applied as shown in Eq. (11), where all power is assumed to be entering the element.

$m_{i, j} c_{p, i, j} \frac{d T_{i, j}}{d t}=\dot{Q}_{c d, i-1, j}+\dot{Q}_{c d, i+1, j}+\dot{Q}_{c d, i, j+1}+\dot{Q}_{c n v, i, j-1}+\dot{Q}_{r d, l}$

Each component shown in Eq. (11) must be defined to accurately represent the heat transfer occurring. The term $d T_{i, j} / d t$ is represented as $\left(T_{i, j}^{t+1}-T_{i, j}^{t}\right) / \Delta t$ and the term $m_{i, j}$ as $2 \pi r_{m} \Delta r \rho$. For the conduction heat transfer, the use of the harmonic mean for the thermal conductivity is implemented rather than the arithmetic mean, since it provides a better representation at an interface between two different materials [24]. The conduction term then simplifies as shown in Eq. (12). In a similar manner, the convection term simplifies as shown in Eq. (13).

$\dot{Q}_{c d, i-1, j}=\frac{\left(2 k_{i-1, j} k_{i, j}\right)\left(2 \pi r_{m} \Delta r\right)}{\left(k_{i-1, j}+k_{i, j}\right) \Delta z}\left(T_{i-1, j}-T_{i, j}\right)$

$\dot{Q}_{c n v \cdot j-1}=h\left(2 \pi r_{i n} \Delta z\right)\left(T_{i, j-1}-T_{i, j}\right)$

where $r_{i n}$ represents the inner diameter which is equal to $r_{c}$ and $r_{m}$ represents the middle diameter which is equal to $r_{i n}+\Delta r / 2$. The only term that still needs to be defined in Eq. (11) is that for the radiation heat transfer. This term heavily relies on the results from the MCRT and it couples both analyses together. The total radiation absorbed by the first layer within the cavity consists of the amount of radiation incident on the element by direct and indirect radiation

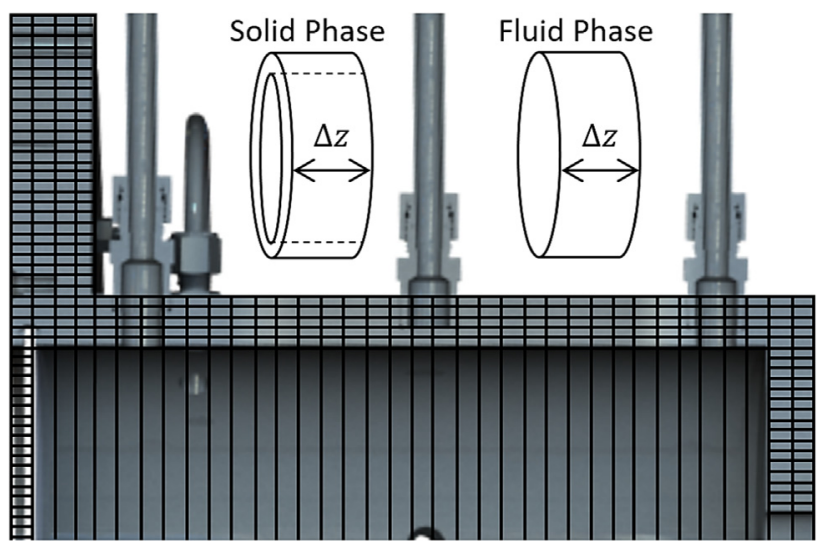

Fig. 7. Solar receiver's heat transfer mesh. 
from the source and other surfaces within the cavity minus that radiated by the surface element $l$. This is represented by Eq. (14), where the $i, j$ terms are substituted with $l, m$ in order to avoid confusing the MCRT surface elements terminology with that of the volumetric heat transfer ones.

$\dot{Q}_{r d, l}=\sum_{m=1}^{n} D_{m, l} \dot{Q}_{m}-\varepsilon_{l}\left(2 \pi r_{i n} \Delta z\right) \sigma T_{l}^{4}$

Just in a similar manner as described above for the first horizontal layer within the cavity, several equations are obtained in order to represent the heat transfer into different volumetric elements at the boundary surfaces of the receiver. At the outer external surfaces of the receiver, it is assumed that the receiver encounters heat losses in the form of convection and radiation losses, where the ambient temperature is assumed to remain constant at $20^{\circ} \mathrm{C}$. The equation then representing the interior elements only consists of conduction through the four surfaces of the element.

On the other hand, for the fluid phase, the mass conservation states that mass entering is the same as that leaving the control volume, which is equal to $\dot{m}$. Therefore, based on conservation of energy for an element in the fluid phase, energy due to fluid flowing in as well as that of heat convection must equal to that of the fluid flowing out. With that mentioned, the equation for the temperature at each location within the fluid phase is simplified and presented in Eq. (15), where $A=2 \pi r_{c} \Delta z$. By now, all equations representing the heat transfer are obtained and are solved numerically in order to provide the temperature within the receiver for both the transient and steady state.

$T_{i, j}=\frac{h A}{\dot{m} c_{p}+h A} T_{i, j+1}+\frac{\dot{m} c_{p}}{\dot{m} c_{p}+h A} T_{i-1, j}$

\section{Results and discussion}

\subsection{Optical analysis}

It is vital to start by checking the accuracy of the optical analysis performed through the MCRT method illustrated earlier. For this, the heat flux distribution on an $80 \times 80 \mathrm{~mm}^{2}$ target is obtained through the model and compared to that obtained using experimental measurements. The experimental three-dimensional heat flux distribution is shown earlier in Fig. 3, where the results of the model exhibits a similar pattern. However, for comparison purposes, a two-dimensional contour on only a $30 \times 30 \mathrm{~mm}^{2}$ portion of the target for 155 A supply current is plotted and shown in Fig. 8 for both the results obtained experimentally and from the model. This comparison is only presented for the maximum supply current value, since any errors are expected to be amplified, making it easier to distinguish errors and discrepancies.

Comparing the experimental and modeled heat flux distribution, it can be noticed that the experimental distribution is not quite as symmetric as expected or as the modeled distribution. The contour is slightly distorted and deformed, which is a behavior often reported for the characterization of solar simulators in previous studies $[23,29,30]$. There are several potential reasons for this slightly distorted shape, which include: the angled position of the CMOS camera, minor alignment errors or shape distortions within the ellipsoidal reflector and solar simulator, and asymmetries in the arc.

For the heat flux distribution obtained from the model, a similar distribution as that of the experimental contour is seen. However, fluctuations are present, which is mainly due to the method of the MCRT. Due to the very fine resolution of the experimental data and that required to capture the distribution of the heat flux, more than 100,000 surfaces are considered at the target. If the value for the
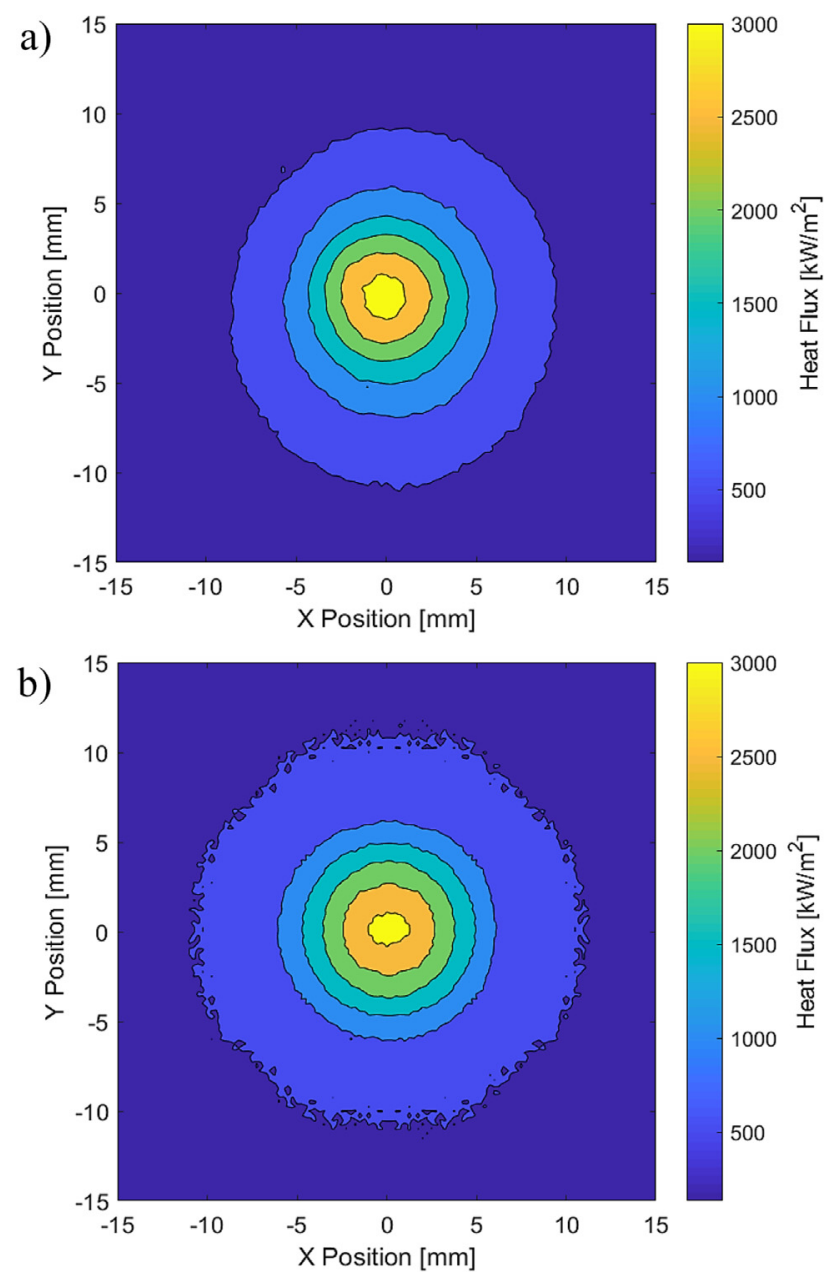

Fig. 8. Heat flux distribution at $f_{2}$ a. experimental, b. model.

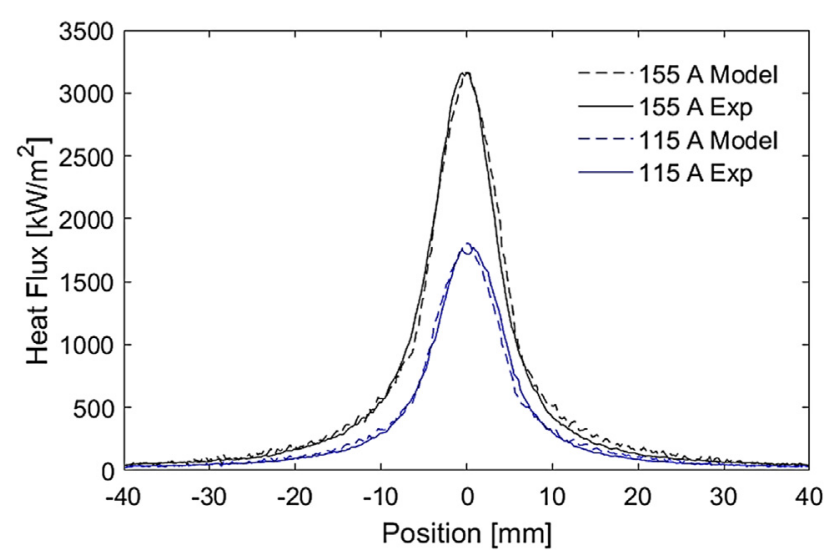

Fig. 9. Two-dimensional heat flux distribution at target center.

number of rays, $N_{i}$, recommended earlier is used, the results from the model would be much smoother. However, this would require a much greater computational time, which comes with an expense. Therefore, around $1 / 20$ of the recommended value was used for the total number of rays. It is noteworthy to state that this issue does not affect the accuracy of the entire model of the receiver, since the total number of surfaces considered throughout the receiver are much lower.

In order to compare the optical analysis from the model further, a two-dimensional heat flux distribution along the center of the 
target is plotted and shown in Fig. 9. This is plotted for the minimum input current value of $115 \mathrm{~A}$ and the maximum value of $155 \mathrm{~A}$. Since the heat flux distribution is taken at the center of the target, Fig. 9 also shows a comparison of the maximum heat flux obtained from the model versus that experimentally measured. The maximum heat flux ranges from 1803 to $3163 \mathrm{~kW} / \mathrm{m}^{2}$ for the model, while the total power intercepted at the second focal point ranges from 566 to $908 \mathrm{~W}$. The half width is calculated to range from 4.65 to $4.75 \mathrm{~mm}$, while half the power ranges from 3.90 to $3.95 \mathrm{~mm}$. These values, when compared to the experimentally obtained values stated earlier, validates the accuracy of the optical analysis, resulting in the radiation source being wellsimulated. Therefore, this should decrease the errors associated with the heat transfer analysis, and hence the entire model.

\subsection{Heat transfer model}

Once the optical analysis has been examined thoroughly and validated, the heat transfer model is then analyzed. The model undergoes several validation measures through the use of experimental results. The first set of experimental validation includes the examination of the transient response of the model. For this, a transient temperature response for the model has been obtained and compared to experimental results at an input current of $155 \mathrm{~A}$ to the solar simulator. Fig. 10a and b show the transient temperature responses for two different volumetric flow rates of 5 and 10 SLPM. These transient responses are with a maximum aperture radius of $60 \mathrm{~mm}$, where the variable aperture was unmounted. The corresponding steady state contours for each are also shown in Fig. 10c and Fig. 10d.
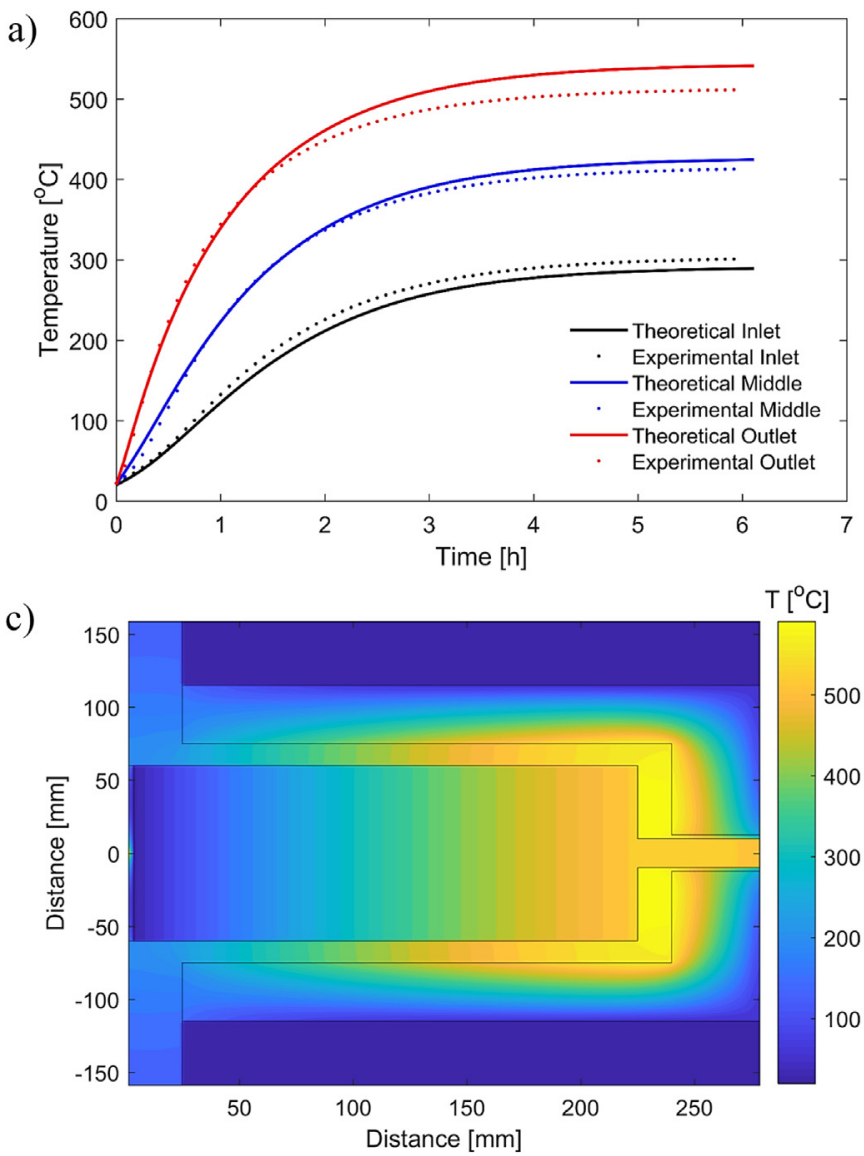

For a volumetric flow rate of 5 SLPM, the maximum percentage differences, which are also the steady state differences, between the experimental and simulated temperatures are 3.9, 2.9, and $5.9 \%$ for the inlet, middle, and outlet temperatures. On the other hand, for the 10 SLPM, the differences are $2.5,2.9$, and $4.7 \%$. These differences are within an acceptable accuracy. Based on the results shown in Fig. 10, it is clear that the model overestimates the temperatures at the middle and outlet of the receiver, while it underestimates the temperatures at the inlet, for both volumetric flow rates. Thus, the model predicts higher power distribution within the rear section of the receiver than it actually is. This incorrect prediction is probably due to one or more of the model's assumptions stated earlier. If the surfaces of the system are treated as nondiffuse non-gray surfaces, the radiation absorbed, emitted, and reflected within the solar receiver will be different than that predicted by the model. In addition, if the refractive index of the quartz window is considered and not assumed to be one, the rays transmitted through the window will be altered and behave differently from that predicted. Therefore, the assumptions taken by the model create a bias in the power distribution towards the rear end of the receiver, which causes higher temperature estimates, with respect to the experimental results, at the back end of the receiver.

From the steady state temperature contours obtained for both volumetric flow rates, shown in Fig. 10, it can be noticed that increasing the flow rate from 5 to 10 SLPM does not create a significantly large decrease in temperature within the solar receiver. Increasing the flow rate by a factor of two only decreased the temperatures by $18.9,17.6$, and $11.2^{\circ} \mathrm{C}$ at the inlet, middle, and outlet, while it decreased by $73.5^{\circ} \mathrm{C}$ for the exhaust fluid. Therefore, adjusting the feedstock's flow rate might not enable for complete
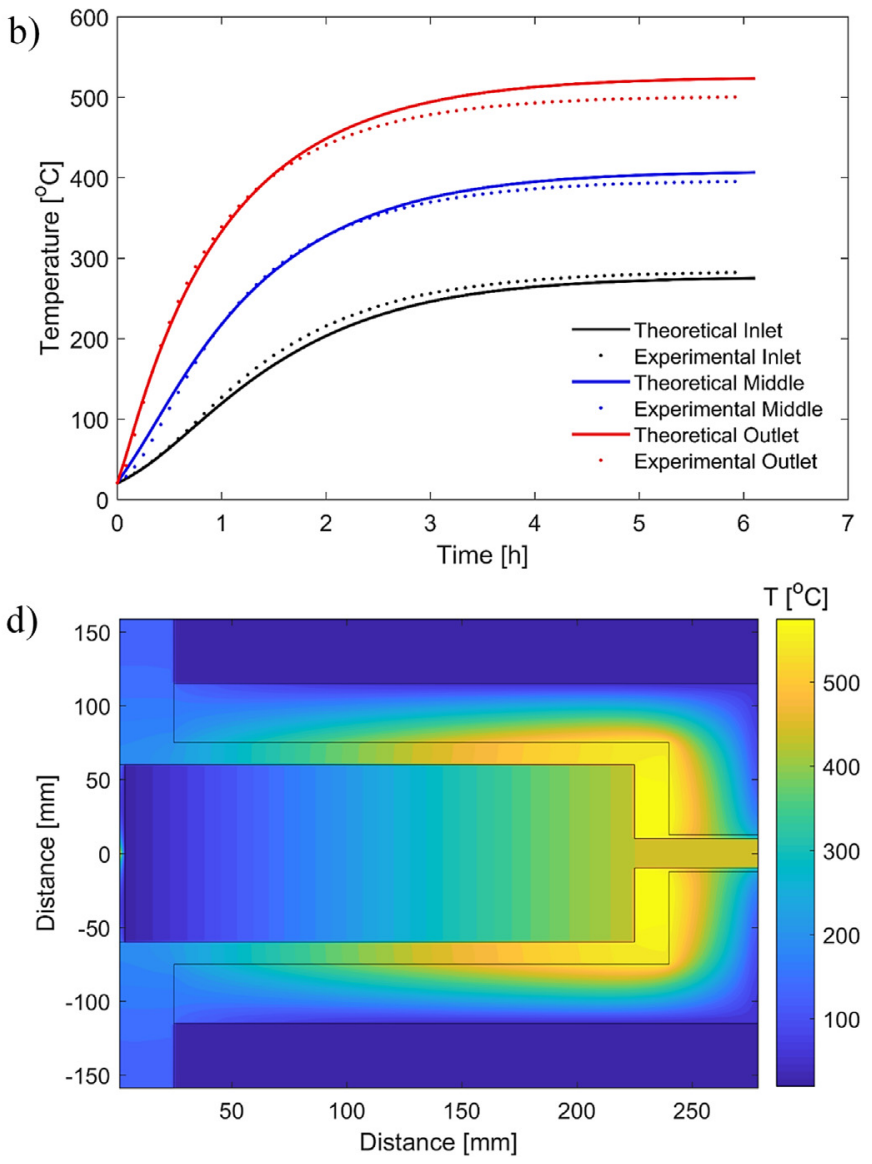

Fig. 10. Transient temperature response for a. 5 SLPM and b. 10 SLPM, steady state temperature contours for c. 5 SLPM and d. 10 SLPM. 
control over temperature within the receiver, as opposed to a variable aperture mechanism, as will be demonstrated later. Solely adjusting the flow rate to regulate temperature within the receiver might not be possible without disturbing the flow pattern. Therefore, the variable aperture seems to be a promising addition to the control methodology for the temperature within a solar receiver.

Furthermore, based on the temperature contours obtained, it can be noticed that there are hotspots present on the back plate of the receiver. This is due to the characteristics of the solar power input from the simulator, where rays from the source are not provided with enough distance through the receiver, from the second focal plane, before hitting the back plate. Hotspots lead to poor heat transfer and overall efficiency of the solar receiver, since the radiation emission increases significantly, and thus, increasing thermal losses. In order to avoid hotspots, the geometry of the receiver can be enhanced. This can be achieved by decreasing the diameter of the receiver's cavity or increasing its total length. This will in turn decrease the probability of rays from the source hitting the back plate and causing severe hotpots. Hence, a more uniform temperature distribution can be obtained. However, hotspots are believed to not be an issue currently, since the implementation of a participating medium, which is the end goal, will scatter and absorb a significant amount of radiation from the source. Therefore, the hotspot issue will reduce significantly, and might no longer exist with a participating medium.

The model is then validated with the aperture being mounted onto the solar receiver. Steady state temperatures were obtained experimentally for an input current of 155 A to the solar simulator and a volumetric flow rate of 10 SLPM for different aperture's radii. Fig. 11 shows how the steady state temperatures at the inlet, middle, and outlet of the receiver vary with respect to the aperture's radius.

Based on the validation performed so far, the numerical model provides results with satisfactory accuracies for the steady state temperature under different conditions. However, from the results shown in Fig. 11, it can be noticed that there is a significantly large difference between the temperatures from the model and that measured experimentally at an aperture radius of $4.5 \mathrm{~cm}$. This is the largest radius where the variable aperture was mounted and tested. There is an average percentage difference of around $13.1 \%$ at that radius. Also, it can be noticed that the trend exhibited by the experimental measurements for an aperture's radii of $4.5 \mathrm{~cm}$ and lower does not lead to that measured for a radius of $6 \mathrm{~cm}$, but rather leads to significantly lower temperature values. This is unlike the response of the results of the model, where both sets of results seem to deviate starting from a radius of $1.5 \mathrm{~cm}$, but converge back again only for the measurement at a radius of $6 \mathrm{~cm}$. This shows the effect that mounting of the variable aperture had on the

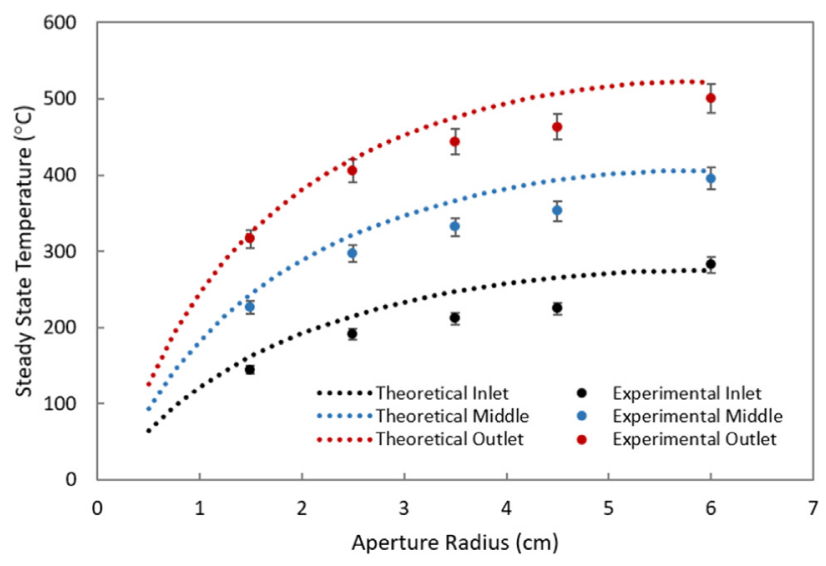

Fig. 11. Steady state temperatures with varied aperture's radii. system. Since the variable aperture has a finite thickness, the opening of the mechanism deviates a little from the receiver's aperture and second focal plane. Hence, blocking some of the power from the source, and significantly affecting that entering the solar receiver. Therefore, less power gets through to the solar receiver, leading to lower experimentally measured temperature values when compared to the ones obtained from the model. Another potential reason for the difference is due to the slight deviation of the variable aperture from a perfect circle.

Fig. 11 illustrates the negative impact that the variable aperture mechanism can have on the performance of the solar receiver. However, the lower its thickness is, the lower the impact it will have on performance. Efforts should be taken to decrease the mechanism's thickness, without compromising its robustness. The ideal case would be to have the variable aperture integrated within the actual receiver, so that both the original aperture and the variable aperture coincide.

Fig. 11 also illustrates an important phenomena, which will be more significant and important when the solar receiver is operating at higher temperatures. By looking into the steady state temperatures response obtained from the model at different locations, it was noticed that the maximum steady state temperatures do not occur at the maximum opening radius of $6 \mathrm{~cm}$. They rather occur at the slightly lower radius value of $5.75 \mathrm{~cm}$, with slightly higher steady state temperatures. These differences, which range from 0.2 to $0.4{ }^{\circ} \mathrm{C}$, are insignificant at the moment, since temperatures within the receiver do not exceed $550{ }^{\circ} \mathrm{C}$. However, at higher temperatures, radiation heat transfer will be much more dominant and these temperature differences will be much greater, as presented by Steinfeld and Schubnell in an earlier study [3]. Hence, through the trends shown in Fig. 11, the variable aperture seems to be a promising addition to solar receivers, whether the goal is to control the temperature within a receiver or obtain the highest achievable one.

\subsection{Uncertainty analysis}

An uncertainty analysis is vital in ensuring that the experimental results obtained and the validation of the model are viable. The analysis, as acquired from the American National Standard Institute (ANSI), categorizes uncertainty into two different types; type $A$, which is the uncertainty due to the effect that the experimental setup has on measurements, and type $B$, which is the uncertainty of the sensors [31]. Eq. (16) is then used to obtain the total uncertainty, given a confidence level of $95 \%$.

$U_{\text {Total }}= \pm 2\left(U_{A}^{2}+U_{B}^{2}\right)^{\frac{1}{2}}$

where $U_{\text {Total }}, U_{A}$, and $U_{B}$ are the total, type A, and type B uncertainties. The maximum type $A$ uncertainty was determined through the fluctuations in the measurements, by calculating the largest percentage difference between the averaged experimental measurements and any of its constituents. It was evaluated to be around $\pm 1.8 \%$ throughout all measurement sets. On the other hand, the maximum type B uncertainty was determined through the maximum uncertainty of the type $\mathrm{K}$ thermocouples used, and was evaluated to be around $\pm 0.4 \%$. Hence, the maximum total uncertainty was calculated to be $\pm 3.7 \%$, with a $95 \%$ confidence level. Based on the uncertainty value calculated, it is determined that most measurements are within the experimental uncertainty or relatively close to it.

\subsection{Application of variable aperture}

In efforts to determine how the variable aperture and solar receiver will behave under real conditions, data for Direct Normal 
Irradiance (DNI) were obtained from the University of Nevada Las Vegas (UNLV). The DNI from the sun is monitored throughout the year by UNLV, where a certain day with severe fluctuations in the DNI was used here. This DNI response is shown in Fig. 12a, and it corresponds to data gathered on January 1st, 2018 [32]. For the purpose of simulating real life conditions for the model, it was assumed that a maximum irradiance of $925 \mathrm{~W} / \mathrm{m}^{2}$ (maximum DNI on a sunny day) corresponds to an input current to the solar simulator of $155 \mathrm{~A}$. This irradiance assumption was made based on the conditions of the solar simulator for which the model has been validated for. It would be unreasonable to use the model to simulate conditions out of its validated range, even though the end goal of the receiver is to operate at much higher irradiance conditions. The in-house code was then used to simulate the response of the solar receiver's variable aperture, with the sole purpose of maintaining a semi-constant temperature of $250{ }^{\circ} \mathrm{C}$ for the exhaust fluid. This was achieved through implementing a simple control algorithm based on logical operators. The operators compared the exhaust temperature to the set temperature and decided whether to increase or decrease the aperture's radius, or just keep it unchanged. Although a temperature of $250{ }^{\circ} \mathrm{C}$ does not allow for useful chemical reactions or decompositions to take place, it was chosen based on the irradiance assumption previously stated along with the DNI obtained for the simulated day. The response of the variable aperture mechanism is shown alongside the DNI in Fig. 12a and alongside the exhaust temperature in Fig. 12b. Based on the response shown in Fig. 12b, the system takes around $1.5 \mathrm{~h}$ to reach the required temperature, where then the variable aperture starts to adjust in order to maintain a constant temperature of $250{ }^{\circ} \mathrm{C}$. This temperature is then maintained for around $5.5 \mathrm{~h}$, where it then starts declining $30 \mathrm{~min}$ before sunset due to the low Direct Normal Irradiance. This simulated response demonstrates the promising effectiveness of the variable aperture in maintaining temperatures within the solar receiver, without disturbing the flow pattern.
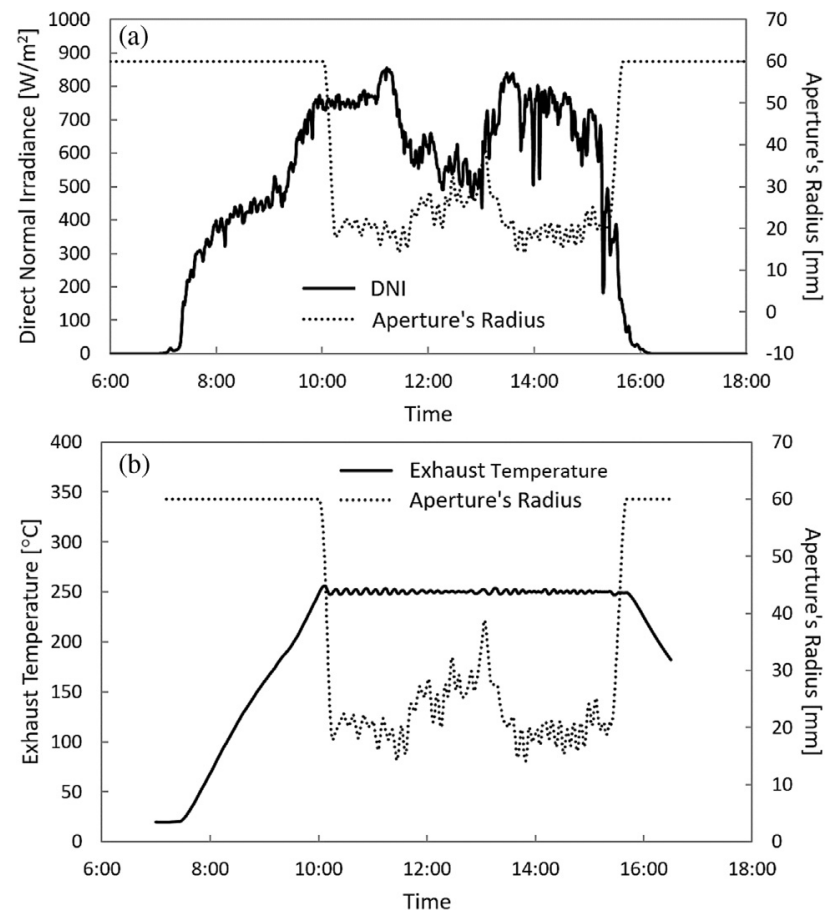

Fig. 12. Variable aperture's response throughout a day with respect to a. DNI, b. exhaust temperature.

\section{Conclusions}

A numerical heat transfer model was presented for a solar receiver with a variable aperture mechanism. The model incorporates an optical analysis performed through the MCRT method to simulate the source irradiance as well as surface radiation emissions and losses. The Xenon arc was approximated by a composite shape consisting of a hemisphere attached to a cylinder, which accurately modeled the arc. The MCRT analysis was then coupled to a heat transfer analysis, performed through the finite volume method, to determine the temperature distribution and heat losses within the receiver. The optical model was validated based on heat flux measurements at the second focal point, where it had absolute percentage errors of approximately $0.8,0.5,1.1$, and $3.2 \%$ for the peak power, total power, half width, and half power. For the heat transfer model, averaged absolute percentage errors of $3.2,2.9$, and $5.3 \%$ at the inlet, middle, and outlet sections of the receiver were determined for different volumetric flow rates. With the variable aperture mechanism mounted onto the solar receiver, the percentage errors increased significantly at the different sections. This was due to the thickness of the mechanism, where its location deviates from the second focal point.

It was determined that a composite shape comprising a hemisphere and a cylinder is capable of accurately modeling the Xenon arc within the solar simulator. Also, it was demonstrated that the variable aperture mechanism is a promising addition to control and regulate the temperature in a solar receiver. The mechanism also seemed to be promising, even in applications were a maximum temperature within a receiver is desired. This was based on an observed optimum aperture radius of $5.75 \mathrm{~cm}$, where a peak in the steady state temperatures occurred. However, this peak was very insignificant (around $0.3^{\circ} \mathrm{C}$ ) at this stage, since the operating temperatures were not high enough for the radiation heat transfer to be dominating. It is believed that this difference will be significant at much higher temperatures. However, with the implementation of the variable aperture, care should be taken to try and minimize the thickness of the mechanism as much as possible, so that the saved radiation power by adjusting the aperture's radius does not cancel out with that blocked by the mechanism. Future work will include further enhancements to the heat transfer model as well as the variable aperture, in addition to implementing a control strategy for the different components of the receiver.

\section{Conflict of interest}

The authors declared that there is no conflict of interest.

\section{Acknowledgements}

The experimental part of this research has been funded by Impulse Fund project \# IMP/14/049 of KU Leuven.

\section{References}

[1] EIA, December 2017 Monthly Energy Review, U.S. Department of Energy, Washington D.C., 2017, p. 2017.

[2] IEA, Technology Roadmap: Solar Thermal Electricity, International Energy Agency, Paris, 2014

[3] A. Steinfeld, M. Schubnell, Optimum aperture size and operating temperature of a solar cavity-receiver, Sol. Energy 50 (1) (1993) 19-25.

[4] Y. Liu, Q. Chen, K. Hu, J.H. Hao, Flow filed optimization for the solar parabolic trough receivers in direct steam generation systems by the variational principle, Int. J. Heat Mass Transf. 102 (2016) 1073-1081.

[5] N. Ozalp, V. Shilapuram, Step-by-step methodology of developing a solar reactor for emission-free generation of hydrogen, Int. J. Hydrogen Energy 35 (2010) 4484-4495

[6] E. Baniasadi, Concurrent hydrogen and water production from brine water based on solar spectrum splitting: process design and thermoeconomic analysis, Renew. Energy 102 (2017) 50-64. 
[7] S. Kraupl, A. Steinfeld, Monte Carlo radiative transfer modeling of a solar chemical reactor for the co-production of zinc and syngas, J. Sol. Energy Eng. 127 (2005) 102-108.

[8] B.G. Lougou, Y. Shuai, H. Xing, Y. Yuan, H. Tan, Thermal performance analysis of solar thermochemical reactor for syngas production, Int. J. Heat Mass Transf. 111 (2017) 410-418.

[9] K.R. Krueger, Design and Characterization of a Concentrating Solar Simulator, PhD thesis, University of Minnesota, Minneapolis, MN, 2012.

[10] D. Hirsch, Hydrogen Production by the Solar Thermal Decomposition of Natural Gas Using a Vortex-flow Solar Reactor, PhD thesis, ETH Zurich, Switzerland, 2003.

[11] J. Petrasch, P. Osch, A. Steinfeld, Dynamics and control of solar thermochemical reactors, Chem. Eng. J. 145 (3) (2009) 362-370.

[12] E. Saade, D. Clough, A.W. Weimer, Model predictive control of a solar-thermal reactor, Sol. Energy 102 (2014) 31-44.

[13] C. Ophoff, N. Ozalp, A novel iris mechanism for solar thermal receivers, J. Sol Energy Eng. 130 (2017) 1-10.

[14] A.K. Menon, A. Farid, N. Ozalp, A new solar reactor aperture mechanism coupled with heat exchanger, Chem. Eng. Trans. 30 (2013) 751 756.

[15] A. Rajan, M. Abouseada, P. Manghaipathy, N. Ozalp, F. Abdul Majid, A. Salem, A Srinivasa, An experimental and analytical study on the feasibility of SMA spring driven actuation of an iris mechanism, Appl. Therm. Eng. 105 (2016) 849-861.

[16] J.R. Howell, R. Siegel, M.P. Mengüç, Thermal Radiation Heat Transfer, fifth ed. CRC Press, 2010, pp. 337-400.

[17] M.F. Modest, Radiative Heat Transfer, third ed., Academic Press, 2013, pp. 247 263.

[18] J.R. Mahan, Radiation Heat Transfer: A Statistical Approach, Wiley, New York 2002, pp. 185-370.

[19] S. Kraupl, A. Steingeld, Monte Carlo radiative transfer modeling of a solar chemical reactor for the co-production of Zinc and Syngas, J. Sol. Energy Eng. 127 (2005) 102-108.

[20] L. Li, C. Chen, A. Singh, N. Rahmatian, N. AuYeung, K. Randhir, R. Mei, J.F. Klausner, D.W. Hahn, J. Petrasch, A transient heat transfer model for high temperature solar thermochemical reactors, Int. J. Hydrogen Energy 41 (2016) 2307-2325.

[21] A.E. Kovtanyuk, N.D. Botkin, K.H. Hoffmann, Numerical simulations of a coupled radiative-conductive heat transfer model using a modified Monte Carlo method, Int. J. Heat Mass Transf. 55 (2012) 649-654.

[22] P.V. Zedtwitz, Hydrogen Production by Solar Thermal Steam Gasification of Coal, ETH Zurich, Switzerland, 2005.

[23] X. Dong, G.J. Nathan, Z. Sun, D. Gu, P.J. Ashman, Concentric multilayer model of the arc in high intensity discharge lamps for solar simulators with experimental validation, Sol. Energy 122 (2015) 291-306.

[24] S.V. Patankar, Numerical Heat Transfer and Fluid Flow, McGraw-Hill, New York, 1980, pp. 30-135.

[25] D. Byun, C. Lee, S.W. Baek, Radiative heat transfer in discretely heated irregular geometry with an absorbing, emitting, and anisotropically scattering medium using combined Monte-Carlo and finite volume method, Int. J. Heat Mass Transf. 47 (2004) 4195-4203.

[26] C. Ophoff, S. Korotunov, N. Ozalp, Optimization of design and process parameters for maximized and stable solar receiver efficiency, in: Proceedings of the 2nd Thermal and Fluid Engineering Conference, ASTFE, Las Vegas, NV, 2017, pp. 1-15.

[27] J. Ballestrin, S. Ulmer, A. Morales, A. Barnes, L.W. Langley, M. Rodriguez, Systematic error in the measurement of very high solar irradiance, Sol. Energy Mater. Sol. Cells 80 (3) (2003) 375-381.

[28] S. Usman, N. Ozalp, Numerical and optical analysis of solar power level adaptable solar reactor, Heat Transf. Eng. 35 (16-17) (2014) 1405-1417.

[29] J. Sarwar, G. Georgakis, R. LaChance, N. Ozalp, Description and characterization of an adjustable flux solar simulator for solar thermal, thermochemical and photovoltaic applications, Sol. Energy 100 (2014) 179-194.

[30] R. Gill, E. Bush, P. Haueter, P. Loutzenhiser, Characterization of a 6 kW highflux solar simulator with an array of xenon arc lamps capable of concentrations of nearly 5000 suns, Rev. Sci. Instrum. 86 (12) (2015) 1-20.

[31] ANSI, American national standard for expressing uncertainty - U.S. guide to the expression of uncertainty in measurement, ANSI/NCSL Z540-2-1997, 1997.

[32] NREL database: solar radiation monitoring for UNLV data, http://midcdmz. nrel.gov/unlv/ (accessed February 15, 2018). 